\title{
Erratum: Exploration of Spin-Dependent Thermoelectricity in the Chiral Double-Stranded DNA Molecule Coupled to Ferromagnetic Leads [Phys. Rev. Applied 12, 024025 (2019)]
}

Lei-Lei Nian, Long Bai, ${ }^{*}$ Wenting Yu, Jun Tang, Huichao Li, Rong Zhang, Rui-Qiang Wang, Xue-Feng Wang, ${ }^{\dagger}$ and $\mathrm{M}$. Wierzbicki

(Q) (Received 16 October 2019; published 5 November 2019)

DOI: 10.1103/PhysRevApplied.12.059901

There is an error in Eq. (13). The correct expression takes the form

$$
S_{\sigma}(\mu, T)=-\left.\frac{\pi^{2} k_{B}^{2}}{3 e} T \frac{\partial \ln \left[T_{\sigma}(\varepsilon)\right]}{\partial \varepsilon}\right|_{\mu} .
$$

The typo does not affect other statements in the paper.

†wxf@suda.edu.cn 